\title{
Chapter 4 \\ Electric Field Modeling for Transcranial \\ Magnetic Stimulation \\ and Electroconvulsive Therapy
}

\author{
Zhi-De Deng, Conor Liston, Faith M. Gunning, Marc J. Dubin, \\ Egill Axfjörð Fridgeirsson, Joseph Lilien, Guido van Wingen, \\ and Jeroen van Waarde
}

\subsection{Introduction}

Major depressive disorder (MDD) is a highly prevalent condition with a lifetime prevalence of nearly $20 \%$ [1]. MDD is currently the second leading cause of disability worldwide, and the World Health Organization (WHO) has predicted that, by

\footnotetext{
Z.-D. Deng $(\square)$

Noninvasive Neuromodulation Unit, Experimental Therapeutics and Pathophysiology Branch, National Institute of Mental Health, National Institutes of Health, Bethesda, MD, USA

Department of Psychiatry and Behavioral Sciences, Duke University School of Medicine, Durham, NC, USA

e-mail: zhi-de.deng@nih.gov

C. Liston

Department of Psychiatry, Weill Cornell Medical College-New York Presbyterian Hospital, New York, NY, USA

Feil Family Brain and Mind Research Institute, Weill Cornell Medical College-New York Presbyterian Hospital, New York, NY, USA

Sackler Institute for Developmental Psychobiology, Weill Cornell Medical College-New York Presbyterian Hospital, New York, NY, USA

F. M. Gunning

Department of Psychiatry, Weill Cornell Medical College-New York Presbyterian Hospital, New York, NY, USA

Institute of Geriatric Psychiatry, Weill Cornell Medical College-New York Presbyterian Hospital, New York, NY, USA

M. J. Dubin

Department of Psychiatry, Weill Cornell Medical College-New York Presbyterian Hospital, New York, NY, USA

Feil Family Brain and Mind Research Institute, Weill Cornell Medical College-New York Presbyterian Hospital, New York, NY, USA
} 
2020, it will be the leading cause of disability. In the Diagnostic and Statistical Manual of Mental Disorders, MDD is also the diagnosis that is most strongly associated with suicide attempts, a phenomenon whose rates have sharply increased over the past two decades in the USA [2]. Present first-line treatment options for MDD include antidepressant medications and cognitive-based therapies. However, a large proportion of patients remain unresponsive to these treatment options [3]. This underscores the urgent need for more personalized approaches to treatments as well as alternative antidepressant therapies, such as noninvasive brain stimulation.

Several noninvasive brain stimulation techniques are now available for the treatment of MDD. Electroconvulsive therapy (ECT) is a highly effective treatment for patients with severe and medication-resistant depression. ECT delivers a series of electrical pulse trains to the brain via scalp electrodes that induce a generalized tonic-clonic seizure in anesthetized patients. For the treatment of MDD in adults, ECT has a sustained response rate of approximately $80 \%$ and a remission rate of $75 \%$ [4]. Despite this superior clinical efficacy, little is known about the interindividual variability in the electric field (E-field) strength and distribution induced by ECT. In this work, we aimed to quantify E-field variability in a depressed patient population and to explore correlates with antidepressant treatment outcome.

Another FDA-cleared treatment for depression is repetitive transcranial magnetic stimulation (rTMS). In depressed patients receiving rTMS, interindividual variability in the induced E-field strength and distribution has not been well characterized. It is not known, for example, what aspect of the E-field is related to improvements in depression symptoms. Such information would be useful for patient selection and/or guide treatment target and dosing.

Conventional magnetic neurostimulation systems use a current-carrying coil to generate a time-varying magnetic field pulse, which in turn produces a spatially varying electric field - via electromagnetic induction - in the central or peripheral nervous system. An alternative approach to generating the time-varying magnetic field is by means of moving permanent magnets. Several systems have been proposed [5-7], involving rotation of high-strength neodymium magnets. One of these systems, termed synchronized transcranial magnetic stimulation (sTMS), was explored as a treatment of depression [8].

The sTMS device is comprised of a configuration of three cylindrical neodymium magnets mounted over the midline frontal polar region, the superior frontal gyrus, and the parietal cortex. The speed of rotation for the magnets was set to the

\author{
E. A. Fridgeirsson · G. van Wingen \\ Department of Psychiatry, University of Amsterdam, Amsterdam, Netherlands \\ J. Lilien \\ Department of Psychiatry and Behavioral Sciences, Duke University School of Medicine, \\ Durham, NC, USA \\ J. van Waarde \\ Department of Psychiatry, Rijnstate Hospital, Arnhem, Netherlands
}


patient's individualized peak alpha frequency of neural oscillations, as obtained by pretreatment electroencephalo-graphy recorded from the prefrontal and occipital regions while the patient remained in an eyes-closed, resting state [9]. The hypothesized mechanism of action is that entrainment of alpha oscillations, via exogenous subthreshold sinusoidal stimulation produced by sTMS, could reset neural oscillators, enhance cortical plasticity, normalize cerebral blood flow, and altogether ameliorate depressive symptoms [10]. In a multi-center, double-blind, sham-controlled trial of sTMS treatment of MDD, there was no difference in efficacy between active and sham in the intent-to-treat sample [8]. No direct electrophysiological evidence of the hypothesized mechanism of sTMS was reported, nor was the stimulation intensity and distribution well characterized. In this work, we evaluate the electric field characteristics of sTMS using the finite element method.

\subsection{Modeling Methods}

\subsubsection{ECT Modeling}

This study included 67 patients who received ECT at Rijnstate Hospital in Arnhem, the Netherlands [11]. ECT was administered using a Thymatron System IV (pulse amplitude $=900 \mathrm{~mA}$; Somatics LLC, Lake Bluff, IL, USA), with bilateral and/or right unilateral electrode placements (see Fig. 4.1). Depression severity was assessed using the Montgomery-Åsberg Depression Rating Scale (MADRS). T1-weighted

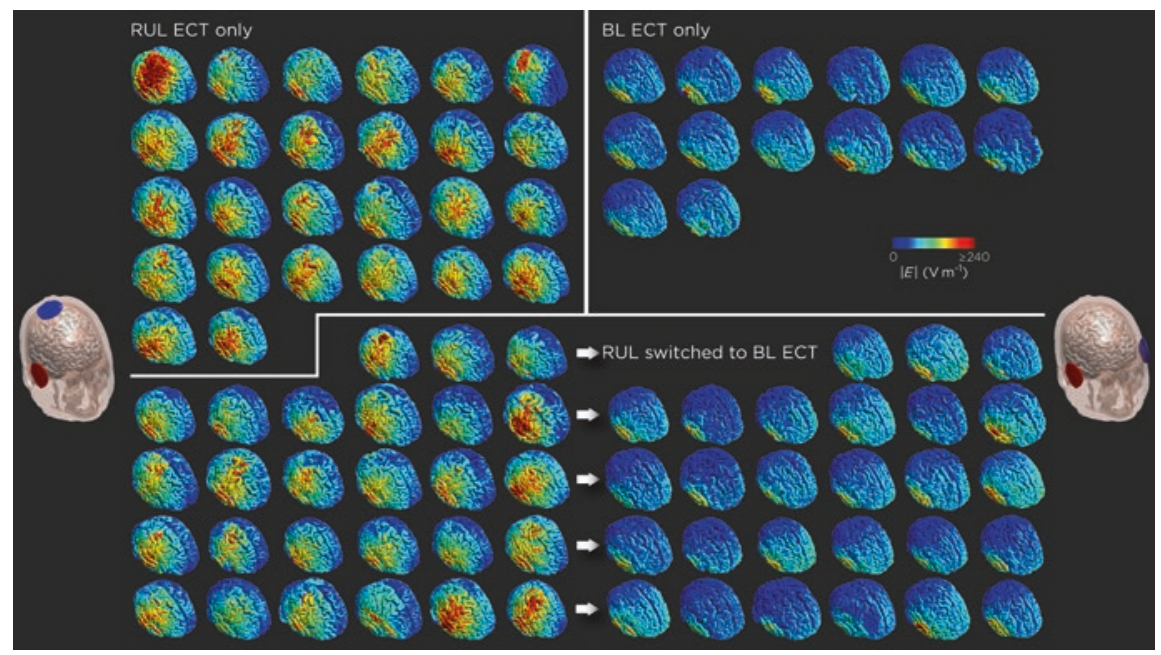

Fig. 4.1 E-field induced in 26 patients receiving right unilateral (RUL) ECT only, 14 patients receiving bilateral (BL) ECT only, and 27 patients who started with RUL ECT and switched to BL ECT 
MRI (1.1 mm isotropic voxel) was acquired at baseline. We examined the relationship between E-field strength and post-treatment MADRS score using a general linear model, controlling for age, sex, baseline MADRS score, and the number of ECT sessions.

\subsection{2 rTMS Modeling}

The Institutional Review Board of Weill Cornell Medical College approved this rTMS study. Twenty-six treatment-resistant depressed patients (age 21-68) participated in the study. Patients received daily $10 \mathrm{~Hz}$ rTMS over the left dorsolateral prefrontal cortex (DLPFC) using the NeuroStar system 5 days per week for 5 weeks [12]. Treatment response was assessed using the 24-item Hamilton Rating Scale for Depression (HAMD-24) at baseline and after the course of rTMS. T1-weighted MRIs were acquired within 7 days prior to starting rTMS and within 3 days of completing the treatment. Diffusion tensor images were acquired using a single-shot spin echo imaging sequence. Motor threshold was determined by visualization of movement technique at baseline. Anatomically realistic finite element head models were constructed from individual MRIs using SimNIBS 2.0.1 [13]. The rTMS coil was centered on the F3 site according to the International 10-20 system [14], oriented 45 degrees toward midline. We evaluated the E-field strength at the DLPFC gray matter (middle frontal gyrus (MFG), inferior frontal sulcus (IFS), and superior frontal sulcus (SFS)).

\subsection{3 sTMS Modeling}

Patient MRI data was not available to model sTMS. The finite element model was implemented in COMSOL Multiphysics (COMSOL, Burlington, MA) using its version of the IEEE Specific Anthropomorphic Mannequin (SAM) phantom as a basis for the geometry (Fig. 4.2). The head model (stator) has uniform, isotropic electrical conductivity of $0.33 \mathrm{~S} \mathrm{~m}^{-1}$ and a relative permeability of 1 . Three cylindrical magnets (rotors) were positioned along the midline: magnet \#1 was located over the frontal pole just above the eyebrows, magnet \#2 was $7.1 \mathrm{~cm}$ from Magnet \#1, approximately overlying the superior frontal gyrus, and magnet \#3 was $9.2 \mathrm{~cm}$ from magnet \#2, approximately overlying the parietal cortex. Each magnet was $2.54 \mathrm{~cm}$ in diameter and height, diametrically magnetized, with a residual flux density of $0.64 \mathrm{~T}$. The axes of rotations were perpendicular to the sagittal plane and the rotation velocity was set to $10 \mathrm{~Hz}$, corresponding to approximately peak alpha frequency. The resulting adaptive mesh consisted of 56,825 tetrahedral elements.

Under the vector potential formulation, Ampère's law was first applied to all domains: 
Fig. 4.2 Dimensions and placement of the three cylindrical magnets in the sTMS system

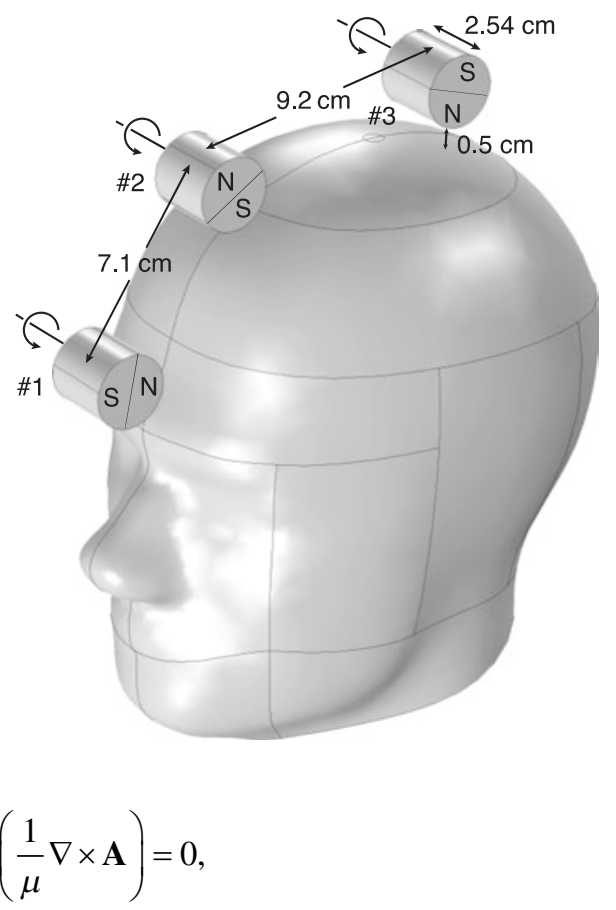

and a magnetic flux conservation equation for the scalar magnetic potential was applied to current-free parts of both the rotor and stator:

$$
-\nabla \cdot\left(\mu \nabla V_{\mathrm{m}}-\mathbf{B}_{\mathrm{r}}\right)=0
$$

Continuity in the scalar magnetic potential was enforced at the interface between the rotor and stator. A stationary solution was first obtained using a direct solver (MUMPS), and then the time-dependent problem (in 10 degrees rotation steps) was solved. This assumes that the transient effects of initiating the rotating magnets have decayed, and the final solution reflects steady-state behavior.

\subsection{Results}

\subsubsection{Electric Field Induced by ECT}

Figure 4.1 shows the E-field distribution induced by bilateral and unilateral ECT in the study patients. For right unilateral ECT, the maximum E-field strength induced in the brain is $513.0 \pm 113.2 \mathrm{~V} \mathrm{~m}^{-1}$. For bilateral ECT, the largest cluster of white matter voxels where the E-field strength is significantly correlated with the posttreatment MADRS score includes parts of the right inferior fronto-occipital 
fasciculus, inferior longitudinal fasciculus, uncinate fasciculus, anterior thalamic radiation, and the corticospinal tract, where there is high E-field strength.

\subsubsection{Electric Field Induced by rTMS}

Figure 4.3 shows the TMS-induced E-field distribution in a representative patient. At the treatment intensity, the mean maximum induced E-field strengths at the MFG, IFG, and SFG are $92.2 \mathrm{~V} \mathrm{~m}^{-1}, 56.5 \mathrm{~V} \mathrm{~m}^{-1}$, and $79.6 \mathrm{~V} \mathrm{~m}^{-1}$, respectively. Stimulator intensity was positively correlated with E-field strength at the MFG

A
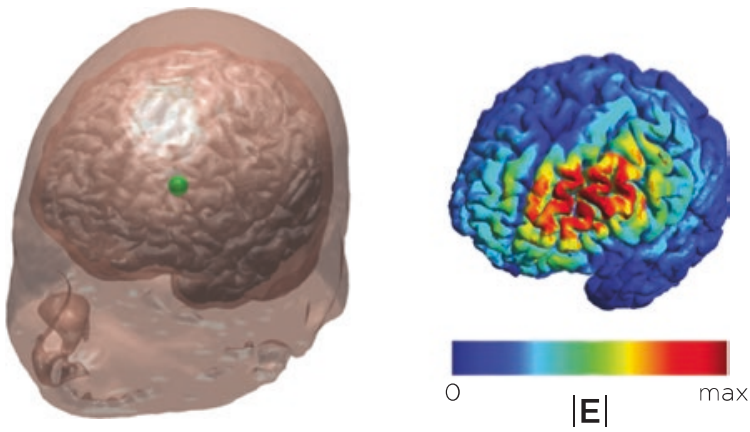

B

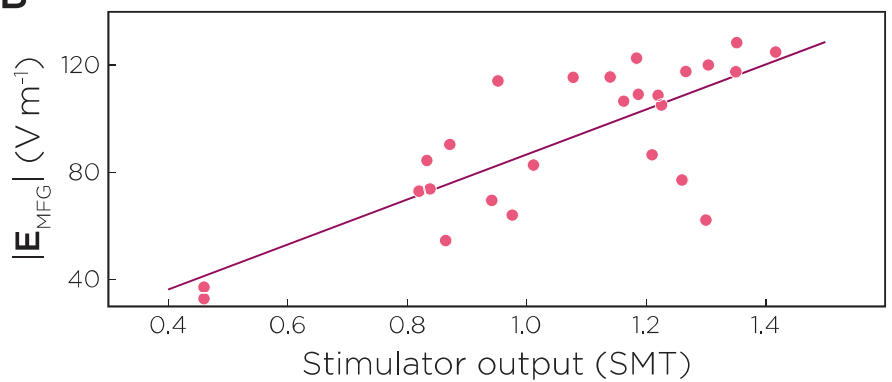

C

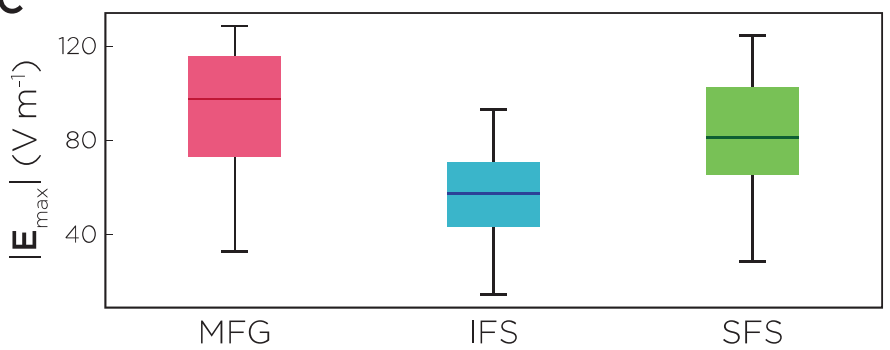

Fig. 4.3 rTMS-induced E-field. (a) Head model and E-field distribution in a representative patient. The green dot on the head model indicates location of the TMS target. (b) Correlation between stimulation intensity (in standardized motor threshold (SMT) units) and maximum E-field strength at MFG. (c) Distribution of E-field strengths at the MFG, IFS, and SFS, for the 26 patients 
( $r=0.77, p<0.001)$. However, E-field strengths at the MFG, IFG, and SFG were not correlated with changes in HAMD-24.

\subsubsection{Electric Field Induced by sTMS}

Figure 4.4 shows the electric field distribution of the full sTMS configuration in the SAM head model. The stimulation is broadly distributed over midline frontal polar, medial frontal, and parietal regions. The peak-induced electric field strength at the surface of the head is approximately $0.06 \mathrm{~V} \mathrm{~m}^{-1}$. At a depth of $1.5 \mathrm{~cm}$ from the head surface, corresponding to the depth of the cortex, the electric field strength attenuates to approximately $0.02 \mathrm{~V} \mathrm{~m}^{-1}$.

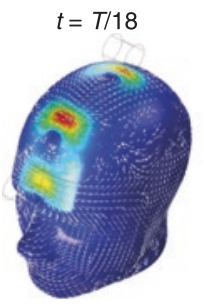

$6 T / 18$

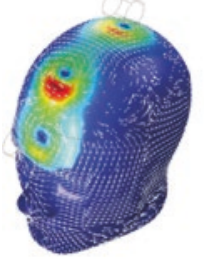

$11 T / 18$

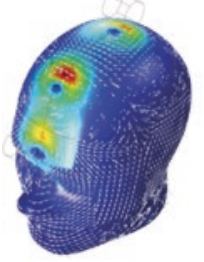

$16 T / 18$

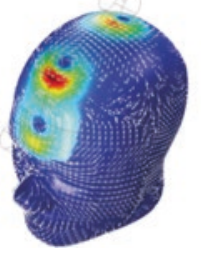

$2 T / 18$

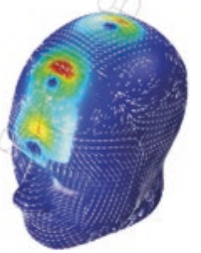

$7 T / 18$

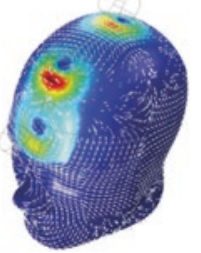

$12 T / 18$

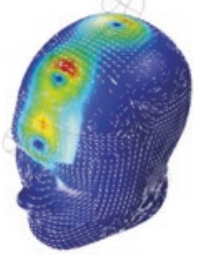

$17 T / 18$

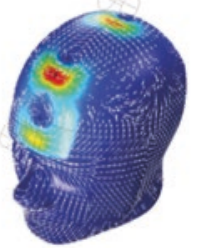

$3 T / 18$

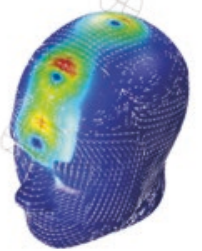

$8 T / 18$

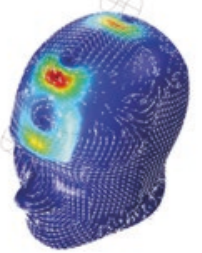

$13 T / 18$

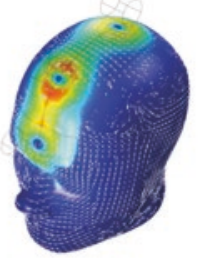

$T$

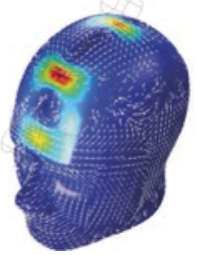

$4 T / 18$

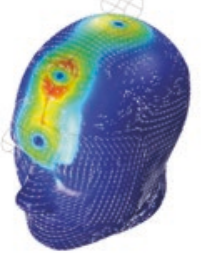

$9 T / 18$

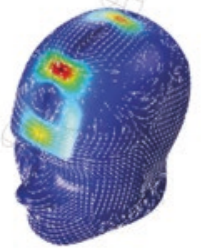

$14 T / 18$
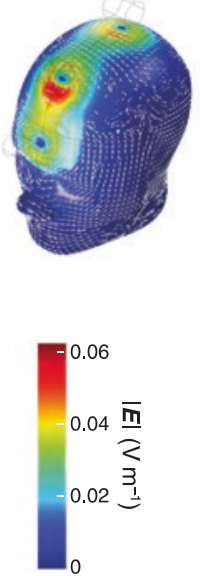

$5 T / 18$

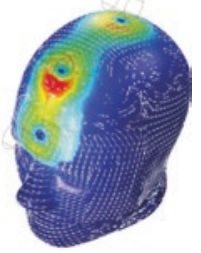

$10 T / 18$

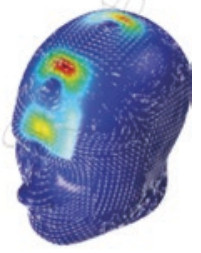

$15 T / 18$

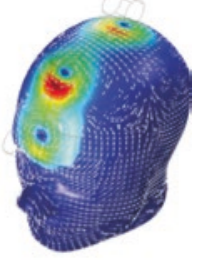

Fig. 4.4 One full revolution (period $=T$ ) of the full sTMS configuration in steady-state 


\subsection{Discussion}

There is marked variability in the distribution of E-field induced by ECT across individuals, with approximately $22 \%$ variation in the maximum E-field strength attributed to anatomical differences. Stimulation of anterior-posterior oriented white matter tracts on the right hemisphere, such as the inferior fronto-occipital fasciculus and inferior longitudinal fasciculus, appears to be related to clinical outcome.

There is also marked variability in the induced E-field strength at the DLPFC in patients receiving rTMS. Region of interest analysis of the E-field distribution in combination with clinical outcome could inform targeting and dosing strategies.

Jin and Phillips estimated the intensity of sTMS stimulation to be approximately $0.1 \%$ that of standard TMS [9]. However, this estimate was based on comparison of maximum surface fields and does not account for boundary conditions of the head. Our simulation with a head model suggests that the peak electric field strength at the level of the cortex is approximately $0.02 \mathrm{~V} \mathrm{~m}^{-1}$. This field strength is an order of magnitude lower compared to those induced by transcranial current stimulation (tCS) [15] and low-field magnetic stimulation (LFMS) [16, 17]. The sTMS field strength is comparable to that of low-intensity repetitive magnetic stimulation (LI-rMS) in an in vitro model, which has been shown to alter cellular activation and gene expression in an organotypic hindbrain explant and in a stimulation frequency-specific manner [18]. Thus, the low field strength of sTMS could be biologically active.

Helekar and Voss proposed a device comprised of an assembly of high-speed rotating cylindrical magnets [7]. These N52 grade magnets are smaller (3/8 inch in height and $1 / 4$ inch in diameter) and have stronger surface field $\left(B_{\mathrm{r}}=1.48 \mathrm{~T}\right) \mathrm{com}$ pared to the sTMS magnets. The magnets are axially magnetized, but the axis of rotation is perpendicular to the axis of the cylinder. The motor provides a no-load speed of 24,000 rpm $(400 \mathrm{~Hz})$. Since the induced electric field strength is proportional to the angular frequency of rotation, higher rotational speed can increase the electric field strength. Helekar and Voss estimated the intensity of their high-speed rotating magnet device to be approximately $6 \%$ that of TMS, based on voltage measurements made with an inductor search coil [19, 20]. However, measurements made in air and without the conductivity boundaries of the head would likely overestimate the electric field strength. Furthermore, smaller magnets have faster field attenuation with distance compared to larger magnets.

Watterson proposed and tested a similar high-speed rotating magnet device for stimulation of muscle nerves [6]. In a series of in vitro experiments on the cane toad sciatic nerve and attached gastrocnemius muscle, Watterson and Nicholson observed that nerve activation was achievable with a rotational frequency of $230 \mathrm{~Hz}$ [21]. The activation of peripheral nerves is thought to be more sensitive to the gradient of the electric field. To maximize the field gradient, Watterson's device employs a "bipole" configuration, comprising two diametrically magnetized cylindrical magnets next to one another with opposite magnetization directions [21].

In this work, we simulated the sTMS system at a fixed rotational frequency of $10 \mathrm{~Hz}$. The frequency of peak alpha oscillation across individuals can vary between 8 and $13 \mathrm{~Hz}$. As mentioned above, the induced electric field strength is proportional 
to the frequency of rotation of the magnets. Therefore, individualizing the rotational frequency could introduce variability in the induced electric field strength across individuals. Higher field strength can be achieved by increasing the rotational speed. However, neuronal activation becomes inefficient at very high frequencies. Finally, the interaction between field strength and excitation frequency could be nonlinear. For example, it has been demonstrated that when $140 \mathrm{~Hz}$ transcranial alternating current stimulation is applied to the motor cortex, low current amplitude of $0.4 \mathrm{~mA}$ results in reduction of motor evoked potential (MEP) amplitudes, intermediate amplitudes of 0.6 and $0.8 \mathrm{~mA}$ showed no effect on MEP, and high amplitude of $1 \mathrm{~mA}$ results in enhancement of MEP amplitudes [22].

\subsection{Conclusion}

We evaluated the electric field characteristics of ECT, rTMS, and the sTMS system of rotating magnets using the finite element method. We found substantial variability in E-field strength across patients receiving ECT and rTMS, possibly contributing to variability in clinical outcome. For the experimental sTMS treatment, we found that the maximum induced electric field strength at the level of the cortex is approximately $0.02 \mathrm{~V} \mathrm{~m}^{-1}$, which is an order of magnitude lower compared to those delivered by transcranial current stimulation and low-field magnetic stimulation. Future work will include simulation of sTMS in anatomically-accurate head models derived from individual brain scans and treatment parameters. Direct electrophysiological data should also be collected to validate the proposed mechanism of action.

Acknowledgments Zhi-De Deng is supported by the NIMH Intramural Research Program and NARSAD Young Investigator Award from the Brain \& Behavior Research Foundation.

\section{References}

1. Ferrari, A. J., et al. (2013). Burden of depressive disorders by country, sex, age, and year: Findings from the global burden of disease study 2010. PLoS Medicine, 10(11), e1001547.

2. Stone, D. M., et al. (2018). Vital signs: Trends in state suicide rates - United States, 19992016 and circumstances contributing to suicide -27 states, 2015. MMWR. Morbidity and Mortality Weekly Report, 67(22), 617-624.

3. Rush, A. J., et al. (2006). Acute and longer-term outcomes in depressed outpatients requiring one or several treatment steps: A STAR*D report, (in eng). The American Journal of Psychiatry, 163(11), 1905-1917.

4. Husain, M. M., et al. (2004). Speed of response and remission in major depressive disorder with acute electroconvulsive therapy (ECT): A Consortium for Research in ECT (CORE) report. The Journal of Clinical Psychiatry, 65(4), 485-491.

5. Phillips, J. W., \& Jin, Y. (2013). Devices and methods of low frequency magnetic stimulation therapy. USA.

6. Watterson, P. A. (2014). Device including moving magnet configurations. 
7. Helekar, S. A., \& Voss, H. U. (2014). Method and apparatus for providing trancranial magnetic stimulation (TMS) to a patient.

8. Leuchter, A. F., et al. (2015). Efficacy and safety of low-field synchronized transcranial magnetic stimulation (sTMS) for treatment of major depression. Brain Stimulation, 8(4), 787-794.

9. Jin, Y., \& Phillips, B. (2014). A pilot study of the use of EEG-based synchronized transcranial magnetic stimulation (sTMS) for treatment of major depression. BMC Psychiatry, 14(1), 13.

10. Leuchter, A. F., Cook, I. A., Jin, Y., \& Phillips, B. (2013). The relationship between brain oscillatory activity and therapeutic effectiveness of transcranial magnetic stimulation in the treatment of major depressive disorder. Frontiers in Human Neuroscience, 7(37).

11. van Waarde, J. A., van Oudheusden, L. J., Verwey, B., Giltay, E. J., \& van der Mast, R. C. (2013). Clinical predictors of seizure threshold in electroconvulsive therapy: A prospective study. European Archives of Psychiatry and Clinical Neuroscience, 263(2), 167-175.

12. Liston, C., et al. (2014). Default mode network mechanisms of transcranial magnetic stimulation in depression. Biological Psychiatry, 76(7), 517-526.

13. Thielscher, A., Antunes, A., \& Saturnino, G. (2015). Field modeling for transcranial magnetic stimulation: A useful tool to understand the physiological effects of TMS? Conference Proceedings IEEE Engineering in Medicine and Biology Society, 222-225.

14. Beam, W., Borckardt, J. J., Reeves, S. T., \& George, M. S. (2009). An efficient and accurate new method for locating the F3 position for prefrontal TMS applications. Brain Stimulation, 2(1), 50-54.

15. Miranda, P. C., Mekonnen, A., Salvador, R., \& Ruffini, G. (2013). The electric field in the cortex during transcranial current stimulation. NeuroImage, 70, 48-58.

16. Rohan, M. L., et al. (2014). Rapid mood-elevating effects of low field magnetic stimulation in depression. Biological Psychiatry, 76(3), 186-193.

17. Wang, B., et al. (2018). Redesigning existing transcranial magnetic stimulation coils to reduce energy: Application to low field magnetic stimulation. Journal of Neural Engineering, 15(3), 036022 .

18. Grehl, S., Martina, D., Goyenvalle, C., Deng, Z.-D., Rodger, J., \& Sherrard, R. M. (2016). In vitro magnetic stimulation: A simple stimulation device to deliver defined low intensity electromagnetic fields. Front Neural Circuits, 10(85).

19. Helekar, S. A., \& Voss, H. U. (2016). Transcranial brain stimulation with rapidly spinning high-field permanent magnets. IEEE Access, 4, 2520-2528.

20. Helekar, S. A., et al. (2018). The strength and spread of the electric field induced by transcranial rotating permanent magnet stimulation in comparison with conventional transcranial magnetic stimulation. Journal of Neuroscience Methods, 309, 153-160.

21. Watterson, P. A., \& Nicholson, G. M. (2016). Nerve-muscle activation by rotating permanent magnet configurations. The Journal of Physiology, 594(7), 1799-1819.

22. Moliadze, V., Atalay, D., Antal, A., \& Paulus, W. (2012). Close to threshold transcranial electrical stimulation preferentially activates inhibitory networks before switching to excitation with higher intensities. Brain Stimulation, 5(4), 505-511.

Open Access This chapter is licensed under the terms of the Creative Commons Attribution 4.0 International License (http://creativecommons.org/licenses/by/4.0/), which permits use, sharing, adaptation, distribution and reproduction in any medium or format, as long as you give appropriate credit to the original author(s) and the source, provide a link to the Creative Commons license and indicate if changes were made.

The images or other third party material in this chapter are included in the chapter's Creative Commons license, unless indicated otherwise in a credit line to the material. If material is not included in the chapter's Creative Commons license and your intended use is not permitted by statutory regulation or exceeds the permitted use, you will need to obtain permission directly from the copyright holder.

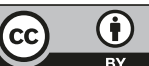

